\title{
Effects of Doping on the Structural Distortion of $\mathrm{La}_{0.7} \mathrm{Dy}_{0.3} \mathrm{Mn}_{1-x} \mathrm{Zn}_{x} \mathrm{O}_{3}$
}

\author{
J.F. LÓPEZ ${ }^{a, *}$, M. RÍOS ${ }^{a, b}$ AND G.A. MENDOZA ${ }^{a}$ \\ ${ }^{a}$ Magnetic Materials and Nanostructures Group, Faculty of Science, Universidad Nacional de Colombia, Carrera 45 \\ No 26-85, Bogotá, Colombia \\ ${ }^{b}$ Department of Basic Science, ECCI University, Bogotá, Colombia
}

\begin{abstract}
Structural properties were studied in the orthorhombic perovskite $\mathrm{La}_{0.7} \mathrm{Dy}_{0.3} \mathrm{Mn}_{1-x} \mathrm{Zn}_{x} \mathrm{O}_{3}$, synthesized in the range $0 \leq x \leq 0.4$. X-ray diffraction data, refined by the Rietveld analysis, shows for $x<0.1$ the presence of stretched octahedra on the Mn-O plane, and, for $x>0.1$, the existence of elongated octahedra along the $c$ axis. Increased $\mathrm{Zn}$ doping causes a direct structural effect: one $\mathrm{Zn}$ atom distorts four neighboring $\mathrm{MnO}_{6}$ octahedra on the $\mathrm{Mn}-\mathrm{O}$ plane. It was observed that the unit cell volume containing Dy decreases as $x$ increases, whereas the unit cell volume containing La increases as $x$ decreases.
\end{abstract}

DOI: 10.12693/APhysPolA.131.1254

PACS/topics: 75.47.Lx, 61.50.-f

\section{Introduction}

The $\mathrm{MnO}_{6}$ octahedron, a basic unit in perovskite manganites, which is a fermion system with a degenerate partially occupied highest molecular orbitals, spontaneously deforms in such a way that the degeneracy is lifted. This phenomenon, known as the Jahn-Teller (JT) effect [1], is a structural phase transition driven by the coupling between the orbital state and the vibronic configuration of the crystal lattice. The JT coupling to the lattice manifests itself in changes of $\mathrm{Mn}-\mathrm{O}_{i}$ bond lengths $L_{i}$ and $\mathrm{Mn}-\mathrm{O}_{i}-\mathrm{Mn}$ angles $\theta_{i}$, as well as in orbital order $[2,3]$. The JT split states appear as a mixing of the degenerate states, which depend parametrically on the JT distortions, i.e., distorted rotated and tilting octahedra that bind to other octahedra with a specific Mn-O-Mn bond angle. Recently, reports indicate that JT-induced orbital polarization effect can lead to orbital ordering in manganite oxides [4]. This novel problem, which relates the orbital texture to the JT effect, requires complete detailed knowledge of the distortion present on these kinds of materials. In this study, $\mathrm{La}_{0.7} \mathrm{Dy}_{0.3} \mathrm{Mn}_{1-x} \mathrm{Zn}_{x} \mathrm{O}_{3}$ was synthesized to study the effect of doping on the structural distortion.

\section{Experimental}

The $\mathrm{La}_{0.7} \mathrm{Dy}_{0.3} \mathrm{Mn}_{1-x} \mathrm{Zn}_{x} \mathrm{O}_{3}$ polycrystalline samples in the doping range $0<x<0.4$ were prepared by solidstate reaction method. In this process, stoichiometric amounts of the precursor reagent $\mathrm{La}_{2} \mathrm{O}_{3}, \mathrm{DyO}_{3}, \mathrm{Mn}_{2} \mathrm{O}_{3}$ $\mathrm{ZnO}_{2}+\mathrm{OH}$ were homogenized and calcined at $1000^{\circ} \mathrm{C}$ for $24 \mathrm{~h}$. X-ray powder diffraction patterns were taken at

*corresponding author; e-mail: room temperature, using a Philips PW1710 diffractometer with copper target through $2 \theta$ step size of $0.02^{\circ}$ with a counting time of $60 \mathrm{~s}$ for each step. The Rietveld refinements were performed using the FullProf software [5] and using pattern files data base COD [6].

\section{Results and discussion}

The observed and calculated diffraction profile obtained from the Rietveld analysis for these samples with $x=0.2$ is shown in Fig. 1(top). All the peaks observed were indexed in three phases: two orthorhombic phases $(P b n m$ and Pnma) and one trigonal phase $(R-3 C)$. The trigonal phase disappeared by calcination at $1100^{\circ} \mathrm{C}$ for $24 \mathrm{~h}$. Bond angles and bond lengths were calculated from the 3D diagrams (Fig. 1(left)). We calculated the samples' structural parameters considering the $\mathrm{LaMnO}_{3}$ as orthorhombic. The results are listed in Table I.

TABLE I

Structural parameters of $\mathrm{La}_{0.7} \mathrm{Dy}_{0.3} \mathrm{Mn}_{1-x} \mathrm{Zn}_{x} \mathrm{O}_{3}$. $n_{1}$ - fraction of orthorhombic phase Pbnm: $\mathrm{LaMn}_{1-x} \mathrm{Zn}_{x} \mathrm{O}_{3}, \quad n_{2}-$ fraction of orthorhombic phase Pnma: $\mathrm{DyMn}_{1-x} \mathrm{Zn}_{x} \mathrm{O}_{3}$

\begin{tabular}{c|c|c|c|c|c}
\hline \hline$x=$ & 0 & 0.1 & 0.2 & 0.3 & 0.4 \\
\hline$\theta_{1}\left[^{\circ}\right]$ & 157.98 & 165.21 & 156.69 & 156.72 & 152.45 \\
$\theta_{2}\left[^{\circ}\right]$ & 157.39 & 150.73 & 147.66 & 145.57 & 146.25 \\
$L_{1}[\AA]$ & 1.9922 & 1.9758 & 1.9870 & 1.9857 & 2.0070 \\
$L_{2}[\AA]$ & 1.9439 & 1.9793 & 2.0114 & 2.0240 & 2.0193 \\
$n_{1}$ & 0.21 & 0.51 & 0.50 & 0.50 & 0.48 \\
$n_{2}$ & 0.25 & 0.25 & 0.23 & 0.24 & 0.22
\end{tabular}

Figure 2 shows $L_{2}>L_{1}$ for $x<0.1$, which implies stretched octahedra, while $L_{1}>L_{2}$ for $x>0.1$ corresponds to elongated octahedra along the $c$ axis. At $x=0.1, L_{1} \approx L_{2}$ implies that the octahedra are nondistorted. 

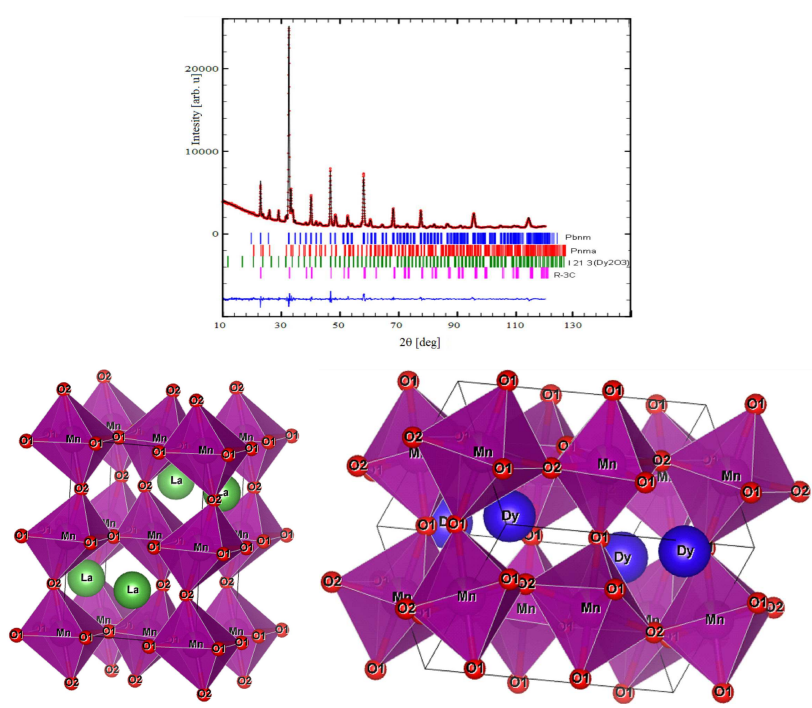

Fig. 1. (top) XRD Rietveld refinement results for $\mathrm{La}_{0.7} \mathrm{Dy}_{0.3} \mathrm{Mn}_{0.8} \mathrm{Zn}_{0.2} \mathrm{O}_{3}$ sample. (left) $\mathrm{LaMn}_{1-x} \mathrm{Zn}_{x} \mathrm{O}_{3}$ orthorhombic structures and (right) $\mathrm{DyMn}_{1-x} \mathrm{Zn}_{x} \mathrm{O}_{3}$ orthorhombic structures.
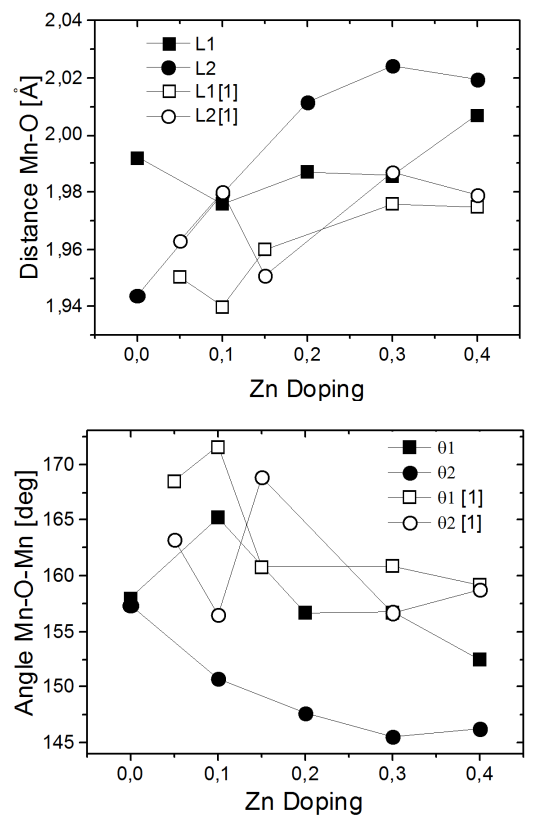

Fig. 2. Structure parameters of $\mathrm{La}_{0.7} \mathrm{Dy}_{0.3} \mathrm{Mn}_{1-x} \mathrm{Zn}_{x} \mathrm{O}_{3}$ (top) $\mathrm{L}_{1}$ (filled squares), $\mathrm{L}_{2}$ (filled circles) compared to bond distances reported (open symbols) [9]. (bottom) Tilting $\theta_{1}$ (a) and tilting $\theta_{2}(\bullet)$ compared to angles reported for $\mathrm{La}_{0.7} \mathrm{Mn}_{1-x} \mathrm{Zn}_{x} \mathrm{O}_{3}$ (open symbols) [9].

TABLE II

Volume of the unit cell.

\begin{tabular}{c|c|c|c}
\hline \hline $\mathrm{XMn}_{1-x} \mathrm{Zn}_{x} \mathrm{O}_{3}$ & \multicolumn{3}{|c}{$V\left[\AA^{3}\right], \mathrm{XMn}_{1-x} \mathrm{Zn}_{x} \mathrm{O}_{3}$} \\
\cline { 2 - 4 } concentration & $\mathrm{La}[9]$ & $\mathrm{La}($ Pbnm $)$ & $\mathrm{Dy}($ Pnma $)$ \\
\hline$x=0.1$ & 233.8 & 235.4 & 226.1 \\
$x=0.2$ & 235.2 & 235.9 & 224.2 \\
$x=0.4$ & 236.6 & 236.21 & 223.1
\end{tabular}

Note, from Table II, that the unit cell volume of the $\mathrm{LaMn}_{1-x} \mathrm{Zn}_{x} \mathrm{O}_{3}(\mathrm{Pbnm})$ phase increases as $x$ increases, agreeing with reports by other authors [7, 8, 10]. This change in the cell volume is expected because the $\mathrm{Zn}$ radius is greater than the $\mathrm{Mn}$ radius.

On the contrary, the unit cell volume in the $\mathrm{DyMn}_{1-x} \mathrm{Zn}_{x} \mathrm{O}_{3}$ (Pnma) orthorhombic phase decreases even though $x$ increases. Given that the atomic radius of Dy is smaller than that of La, we suggest that the Dy allows the enlarged octahedra (by the $\mathrm{Zn}$ inclusion) to tilt into the cell, reducing the net volume of the cell.

\section{Conclusions}

$\mathrm{La}_{0.7} \mathrm{Dy}_{0.3} \mathrm{Mn}_{1-x} \mathrm{Zn}_{x} \mathrm{O}_{3}$ was synthesized in the range $0<x<0.4$. Change in bond length and bond angles, depending on $x$, can be attributed to a distortion of four neighboring octahedra on the $\mathrm{Mn}-\mathrm{O}$ plane by one $\mathrm{Zn}^{2+}$.

The relationship between $L_{2}$ and $L_{1}$ shows two regions: the first for $x<0.1$, where stretched octahedra are present; the second for $x>0.1$, where elongated octahedra along the $c$ axis are present. For $x=0.1$, the octahedra are non-distorted.

The presence of Dy allows increasing the octahedra tilting, reducing the cell volume.

\section{Acknowledgments}

This work was carried out within research project No. 35419 funded by the Faculty of Science at Universidad Nacional de Colombia.

\section{References}

[1] I.B. Bersuker, The Jahn-Teller Effect, Cambridge University Press, Cambridge 2006.

[2] B.B. Van Aken, O.D. Jurchescu, A. Meetsma, Y. Tomioka, Y. Tokura, T.T.M. Palstra, Phys. Rev. Lett. 90, 066403 (2003).

[3] X. Qiu, Th. Proffen, J.F. Mitchell, S.J.L. Billinge, Phys. Rev. Lett. 94, 177203 (2005).

[4] E. Paravini, E. Koch, Phys. Rev. Lett. 104, 086402 (2010).

[5] J. Rodríguez-Carvajal, Physica B 192, 55 (1993).

[6] Cristallography open Database [online].

[7] Sujun Xua, Wei Tong, Jiyu Fana, Jie Gao, Chaozheng Zha, Yuheng Zhang, J. Magn. Magn. Mater. 288, 92 (2005).

[8] Fei Gong, Wei Tong, Shun Tan, Yuheng Zhang, Phys. Rev. B 68, 174410 (2003).

[9] Song Yue, Yuheng Zhang Shun Tan, Phys. Lett. A 319, 530 (2003).

[10] I. Álvarez-Serrano, C. Pico, M.L. Veiga, Solid State Sci. 6, 1321 (2004). 